\title{
A Life Quality Architectural Approach to Enhance Digital Skills Training
}

\author{
Iuliana, I., Marin*, Dorina D. Popovici \\ University POLITEHNICA of Bucharest, Bucharest, Romania \\ Foteini, F., Grivokostopoulou, Constantinos, C., Koutsojannis \\ University of Patras, Patras, Greece \\ marin.iulliana25@gmail.com, dorina.popovici25@gmail.com, grivokwst@ceid.upatras.gr, \\ ckoutsog@upatras.gr
}

The paper presents the demand for professional services and skills in the health sector. The Erasmus+ DIGI4ME project aims to enhance digital skills trainings. An analysis of the Romanian health information system was conducted based on the interviews of the National Public Health Institute of Romania with stakeholders. According to the incidents which occurred in hospitals around the world, a system based on sensors is proposed to mitigate the problems which can appear. The testing of the system was done in laboratory conditions and the reports display the evolution of the measured environmental parameters. The software solution can determine medical professionals to enhance their digital skills and trigger creative ideas to improve the experience of their patients.

CCS CONCEPTS • Applied computing $\rightarrow$ Life and medical sciences; Health informatics; ・ Information systems $\rightarrow$ Information systems applications; Process control systems; Data mining.

Additional Keywords and Phrases: Healthcare, Learning, Electronic system, Digital Skills

\section{ACM Reference Format:}

Iuliana, I., Marin, Dorina, D., Popovici, Foteini, F., Grivokostopoulou, Constantinos, C., Koutsojannis. 2021. A Life Quality Architectural Approach to Enhance Digital Skills Training. In LIQUE 2021: Life Improvement in Quality by Ubiquitous Experiences Workshop, together with IMX 2021: ACM International Conference on Interactive Media Experiences. June 2123, 2021. NY, USA, 4 pages.

\section{INTRODUCTION}

The demographic shifts throughout Europe, will increase the demand for healthcare services and will lead to more jobs, as well as multiple required skills for professionals from the health sector. Health sector will have a $23 \%$ increase in employment by 2025 , reaching even $34 \%$ in Romania [1]. Moreover, the skills that health professionals possess are key aspects for the delivery of high quality services to society. The quality digital skills of health care professionals are acknowledged to be of extreme importance at European Union (EU) level. According to World Economic Forum [2], the health sector will adopt technology by including Internet of Things and connected devices, Big data analytics, artificial intelligence, text, image and voice processing. In this context, a course of introductory programming was taught in 2020 via the Zoom web conference application to health science students from Brazil and the feedback was positive [3]. In Romania, medicine universities have in their curricula a course of medical computing and biostatistics. Kunkle et al. outlined the importance of

* Assistant professor at University Politehnica of Bucharest, Romania.

Published in accordance with the terms of the Creative Commons Attribution 4.0 International Public License (CC BY 4.0). Permission to reproduce or distribute this work, in part or in whole, verbatim, adapted, or remixed, is granted without fee, provided that the appropriate credits are given to the original work, not implying any endorsement by the authors or by SBC. 2021 Brazilian Computing Society. 
incorporating computer science into the training of public health professionals and they should be aware of the implications of Big Data for health [4]. The current COVID-19 pandemic determined the occurrence of opportunities to work remotely and the digitalization of work processes was accelerated. The emerged skills are active learning, creativity, originality, reasoning, user experience, technology use, monitoring and control. In this context, the aim of the DIGI4ME project is to improve digital skills trainings in the health care sector. Educational institutes, health care associations, research institutes and enterprises will exchange abilities, experience and accessibility to be exemplified in a high quality training framework improving digital skill training all over Europe.

In the next section are presented the general overview of the DIGI4ME project, along with a software system which aims to enhance life quality based on sensor usage. Section 3 describes the SWOT analysis of the DIGI4ME project and the test of the proposed system. The last section outlines the conclusions.

\section{METHODOLOGY}

From the beginning of the DIGI4ME project, the educational content will be created by deciding the digital skills which every health professional should have to utilize e-health solutions to their maximum capacity after the check of the specific educational needs of doctors, operators and professionals of digital technology and medical imaging equipment. User-adapted training modules will be created on specific setting regarding digital image processing and administration, including ongoing changes on pedagogical landscape of health care distance learning with the utilization of innovative Vocational Open Online Courses (VOOCs) for digital skills in the health domain. After the assessment of the methodology's usefulness through the pilot study, the quality of national and European Education on digital technologies in health sector will be improved.

An increase of oxygen level to up to $24 \%$ can lead to a fire hazard, like in the case of the Piatra Neamt hospital, Romania; or other locations like Alexandria, Egypt; Chelyabinsk, Russia; Gaziantep, Turkey [5]. In this context, a system to improve life quality was created, because incidents can appear inside hospitals. Due to this reason, sensors should exist inside hospitals to monitor the environment, as well as patients should dispose of panic buttons. The high-level architecture of the system is illustrated in Figure 1.

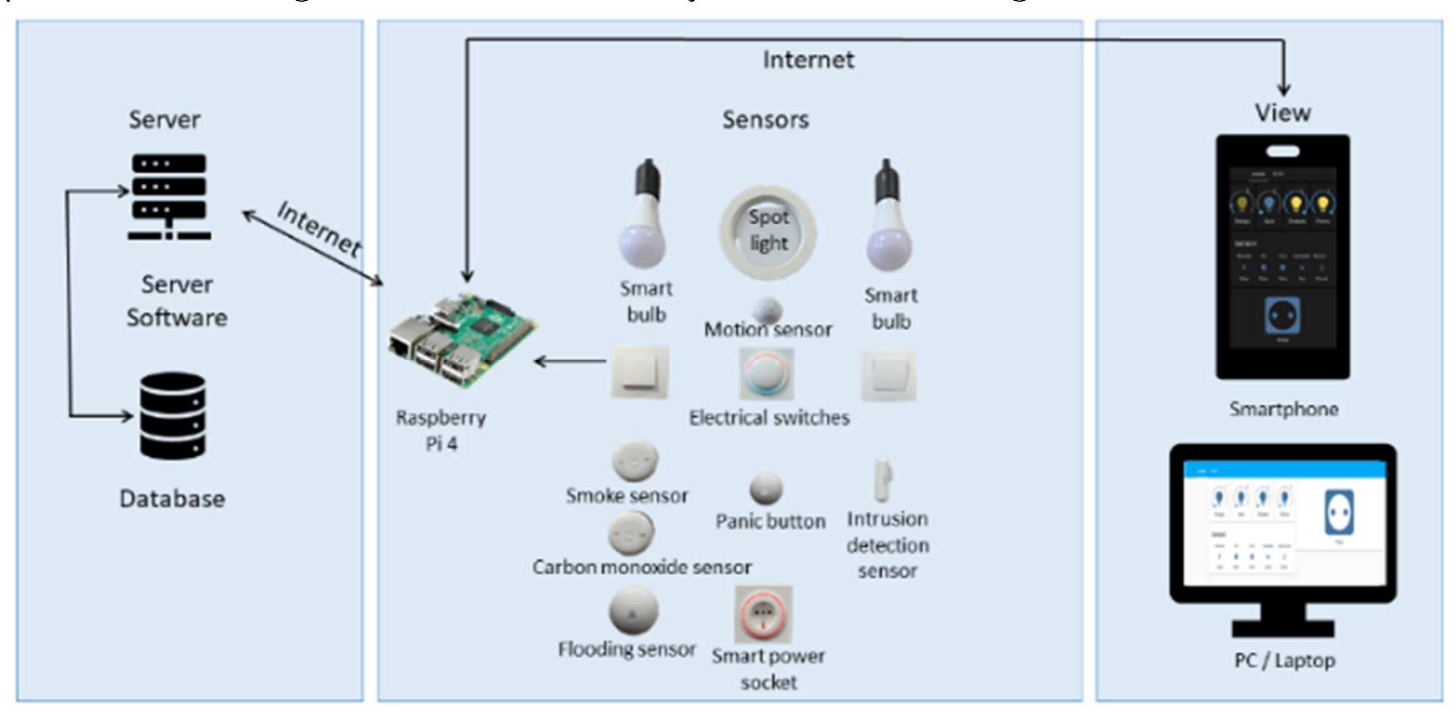

Figure 1: High-level system architecture 
The system employs several types of sensors, namely smart bulbs; spotlight; motion sensor; electrical switches; smoke, carbon monoxide and flooding sensors; panic button; intrusion detection sensor and smart power socket. The data from sensors are sent to the system's gateway, a Raspberry Pi 4 embedded single board computer, via the Z-Wave and Zigbee communication protocols. The server software application securely stores user data, as well as data from his/her environment sensors. The user can access and manage the system via the interface which is available for smartphone, personal computer and laptop.

\section{RESULTS}

A comprehensive Romanian Health Information System (HIS) assessment was conducted based on interviews that were organized by the National Public Health Institute of Romania with stakeholders [6]. After analyzing the assessment results, our observations are reflected in a strengths, weaknesses, opportunities, and threats (SWOT) analysis which are illustrated in Figure 2.

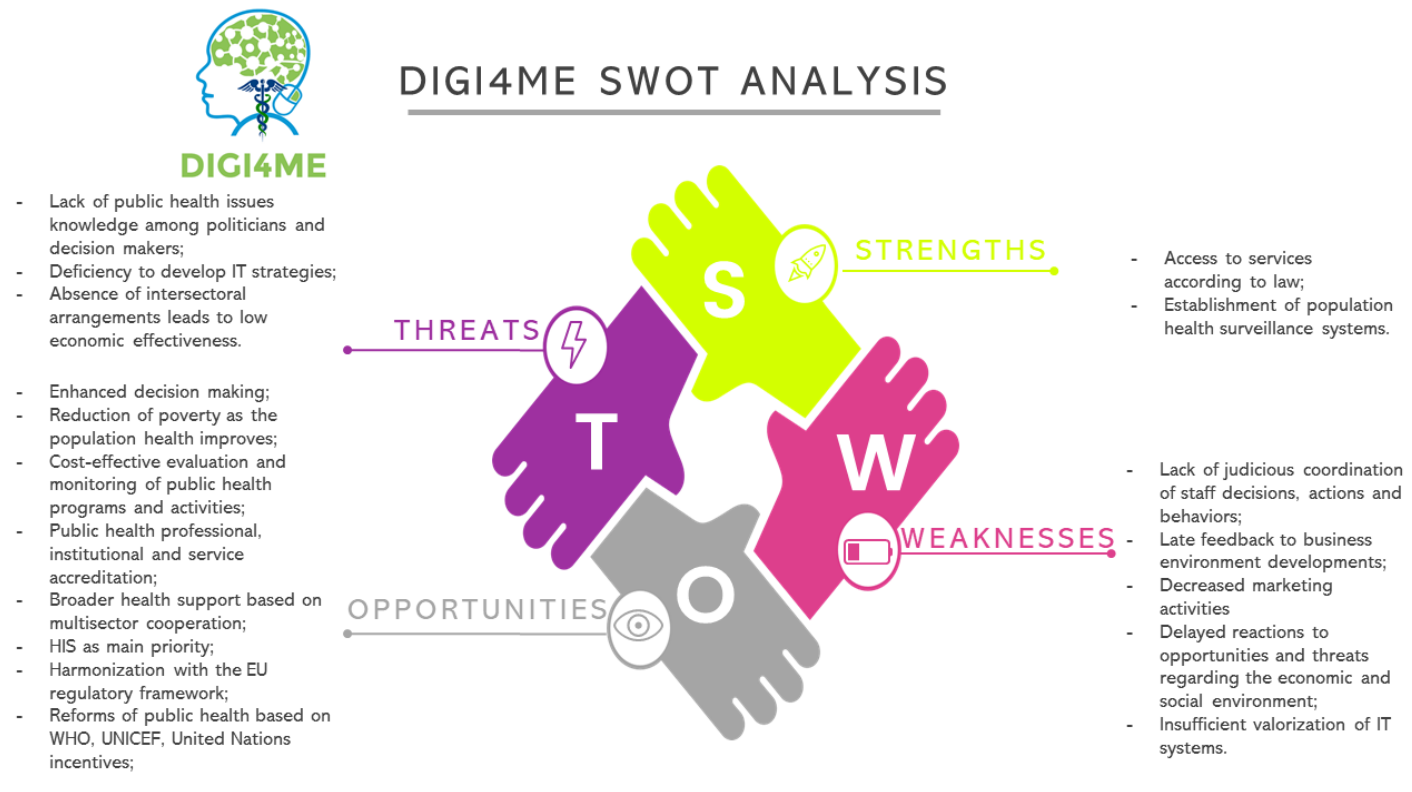

Figure 2: DIGI4ME SWOT analysis for Romania

In the short term, the Romanian HIS ought to extend the capacity of data gathering, processing, examination and reporting system in existent information systems, along with the utilization of data and information based on policies. Classifications for medical care units with beds, outpatient providers, clinical research centers ought to be used at national public level in order to connect distinctive data. In a short to medium term, Romanian HIS ought to urge capable structures to create proficient and sufficient IT solutions in monitoring the results of national health programs.

The proposed system was tested in laboratory conditions, as in Figure 3. The first report contains the type of event (motion detection, light bulb / panel switch off, call closure for mobile devices associated with the application) and the sensor to which it is connected. The time period can be selected for this report. 

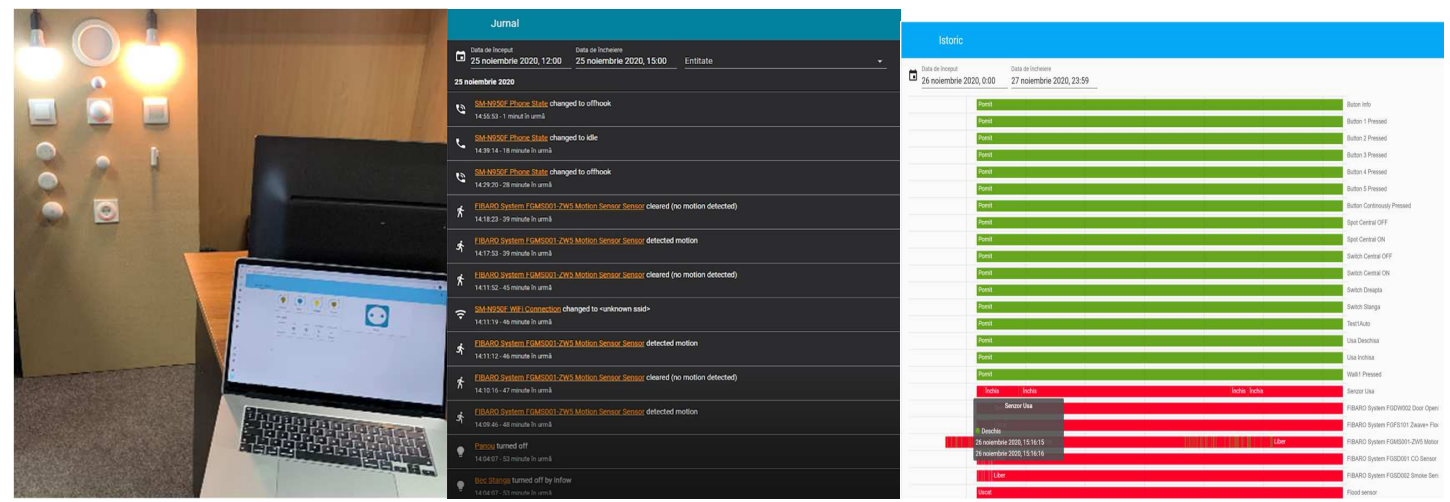

Figure 3: System testing in laboratory conditions. Event report. Sensor report

The second report regards the status of the system sensors. Similar to the event report, the time period can be selected. If the color of a sensor green, it means that the status is on. If the color is red, then the status is closed and the sensor is off. This report is important to determine when it is necessary for alerts to be sent to the patient's smartphone. Further on, the system will be evaluated and improved based on the feedback received from the project's patients and medical staff.

\section{CONCLUSIONS}

DIGI4ME will provide the created open-access training framework, also with its instructive support to the community of health care experts of European Union. The partnership will pursue towards the educational framework to be utilized and integrated in national and European Union level training systems in the healthcare domain. The proposed system can be useful in hospitals, mostly during the current pandemic times and can determine medical professionals to develop their digital skills by using and improving it based on their needs.

\section{ACKNOWLEDGMENTS}

This work was funded by a grant of Erasmus+ A2: Platforms, Skills and Innovation, project number 400621673/2020, DIGI4ME - "Health sector skill alliance for creating innovative and efficient VET programmes and improving the digital skills of medical physics and health professionals".

\section{REFERENCES}

[1] Analytical Highlight: Focus on Health Sector. European Commission. Retrieved December 20, 2020 from https://skillspanorama.cedefop. europa.eu/sites/default/files/EUSP_AH_Health_0.pdf.

[2] The Future of Jobs Report 2020. World Economic Forum. Retrieved January 10, 2021 from http://www3.weforum.org/docs/WEF_Future_ of_Jobs_2020.pdf.

[3] Carlos I. De la Fuente, Eliane Celina Guadagnin, Marcos Roberto Kunzler, and Felipe P. Carpes. 2021. Programming Course for Health Science as a Strategy to Engage Students during the Coronavirus Pandemic. Adv Physiol Educ, 45, 1 (January 2021 ), $53-58$. https://doi.org/10.1152/advan.00183.2020.

[4] Sarah Kunkle, Gillian Christie, Derek Yach, and Abdulrahman El-Sayed. 2016. The Importance of Computer Science for Public Health Training: An Opportunity and Call to Action. JMIR Public Health and Surveillance, 2,1 (March 2016). https://doi.org/10.2196/public health.5018.

[5] Risk of Oxygen-Related Fires in Hospitals Treating COVID-19 Patients. European Commission. Retrieved from February 1, 2021 from https://minerva.jrc.ec.europa.eu/en/shorturl/minerva/llb_2_covidoxygenv2pdf.

[6] Health Information System in Romania: Assessment of HIS in Romania, March 6-7th, 2019. Institutul Național de Sănătate Publică. Retrieved December 10, 2020 from https://insp.gov.ro/sites/cnepss/wp-content/uploads/2019/10/HIS_Romania_Final.pdf?cv=1. 